C2008 IEEE. Personal use of this material is permitted. However, permission to reprint/republish this material for advertising or promotional purposes or for creating new collective works for resale or redistribution to servers or lists, or to reuse any copyrighted component of this work in other works must be obtained from the IEEE. 


\title{
Determining the Level of Perceived Risk in e-business Web 2.0 Interactions
}

\author{
Omar Hussain, Elizabeth Chang, Tharam Dillon and Farookh Hussain \\ Digital Ecosystems and Business Intelligence Institute \\ Curtin University of Technology, Perth, Australia \\ o.hussain@cbs.curtin.edu.au
}

\begin{abstract}
In the domain of e-commerce business it is important for the initiating agent of the interaction to analyze and consider the level of perceived risk in forming an interaction with an agent, apart from just considering the level of trust and security. By doing so, the initiating agent would have all the concepts which would assist it in making an informed interactionbased decision. But for risk analysis in e-business, it is important that the initiating agent considers and assesses it according to its object of analysis in that domain. In our previous work, we have identified the sub-categories of perceived risk in the domain of ebusiness interactions and proposed methodologies by which the initiating agent can quantify them. In this paper, we propose a novel approach by which the initiating agent determines the numerical level and magnitude of perceived risk by utilizing its determined sub-categories in forming an interaction with an agent.
\end{abstract}

\section{Introduction}

The development of the Internet and its integration with Web 2.0 has provided users with sophisticated technologies which ease the process of carrying out their activities. It has enabled the users to collaborate and interact with each other seamlessly across various domains. In this paper, our area of focus is on such architectures that help to form collaborations between any two agents by facilitating an interaction between them, in the domain of Web 2.0 e-business architecture. We aim to highlight the importance and significance of assessing and considering perceived risk within such domain while making an informed interaction-based decision, and then propose a methodology by which the initiating agent can quantify the numeric level and magnitude of perceived risk in it.

The development and advancement of the internet has enabled the users to complete their tasks in less time and reducing the delays associated with the conventional method of interactions. At the same time it has boosted their efficiency and has helped the businesses to improve their sales, productivity and economy. But as mentioned by Chang et al. [1] 'The dynamic, open and convenient web environment not only boosts business potential and the economy but also creates concerns of security, trust, privacy and risks'. So the users, before utilizing the provided facilities to their advantage, should consider and analyze these aspects in order to make sure that they achieve what they desire, or get the maximum output in their interactions. Added to the fact, Web 2.0 is mainly about the people or companies that populate the interaction infrastructure [2]. And when an interaction is being carried out in such an environment, it is up to the interacting entity to make sure that it proceeds in an interaction with that agent or entity in which it hopes to achieve maximum benefit and interaction experience, as the agent in such environments has to make its own decisions. But in spite of such characteristics, the demand of interactions to be carried out in such domain is growing because of their robust nature. According to a report from the Australian Bureau of Statistics updated in 2006, the number of Australian consumers who accepted online shopping in the year 2000 grew to a mammoth $66 \%$ compared with the figures from the previous year when 1.3 million Australians ordered or purchased goods over the Internet in the year [3]. The increasing demand for internet shopping is not limited only to the Internet users in Australia. According to the Dutch home shopping group, in the year 2006 Dutch nationals bought goods worth 4 billion dollars $(2.92$ billion Euros) via the internet, an increase of $28 \%$ from the figures of the previous year [4]. A report by Forrester research showed that in 2003 about $40 \%$ of online European consumers actually purchased online, up from $19 \%$ in 2000 , and in 2006 online shopping in Europe was on the increase, especially in countries like Sweden and Netherlands [5]. But apart from all the advantages provided by such architectures which results in their fast adoption, some important considerations for the users in such interactions are the notions of 'security', 'trust' and 'perceived risk'- in 
other words, those factors which help to make the interactions over this paradigm safe, secure and informed. This is because as mentioned earlier, an agent in such environments has to make its' own decisions and has to be responsive and proactive when doing so.

By doing a review of the literature, it can be concluded that a lot of work has been done by researchers in the area of security and trust, and have developed methodologies by which the initiating agent can assess and consider it while making an informed interaction-based decision. But at the same time, it can be concluded that the concept of perceived risk has widely been ignored in acknowledging it as an important concept for decision-making in business interactions. In this paper, we will highlight the importance of assessing and considering the perceived risk in business interactions while decision-making, and then extend our previous work to ascertain the different levels and magnitude of perceived risk in an e-business interaction. We term the two agents which form an interaction as the 'risk assessing agent' and 'risk assessed agent'. The risk assessing agent is defined as that agent who wants to achieve some desired outcomes in the interaction and initiates an interaction with the other agent. The risk assessed agent is defined as that agent who has the capability to give the risk assessing agent its desired outcomes in the interaction. It is highly possible that there might be more than one risk assessed agent before initiating an interaction and the risk assessing agent while decision making has to decide on which agent to interact with.

In the next section we will highlight the importance of assessing and considering perceived risk in an interaction while decision-making. We then give a brief overview of our previous work in which we define perceived risk according to its object of analysis in e-business interactions and then propose methodologies by which the risk assessing agent quantifies the subcategories of perceived risk. In Sections 3-4 we then propose an approach by which the risk assessing agent utilizes the determined subcategories and ascertains the numeric level and magnitude of perceived risk in forming an interaction with the other agent. We explain the proposed methodology with an example in Section 5 and finally in Section 6 we conclude the paper.

\section{Related Work}

Analyzing and considering the level of perceived risk in an interaction before decision-making is important for the interaction initiating agent as it highlights and expresses the level and degree of loss that can be experienced by it as a result of interaction with the other agent. This level and degree of loss expressed by perceived risk cannot be substituted for by determining either the level of trust or security in the interaction. There is a lot of work in the literature which assesses the level of trust in an interaction and based on that proceeds in decision making [6-10]. In other words those approaches consider trust to be the authoritative concept to risk, and that based only on the level of trust the decision making process in the business interaction can be carried out as it also represents or nullifies the level of risk in the interaction. But trust and risk represent different concepts depending on the different areas that are targeted. In generic terms, trust in the context of business interaction, shows the level of belief that an entity has in the other entity or business, but this level of belief does not express the degree and the magnitude of loss in the interaction. Such concepts can be determined only by the analysis of risk in the interaction in that context. Considering a practical instance from our daily lives, before investing our resources in interacting with an agent or entity within a virtual environment, it is logical to consider questions like:

- To what extent can I rely on the other agent?

- Will the other agent be able to deliver what I want?

- Will I lose the resources that I am investing?

- To what degree will the other agent not deliver what I want?

- What will be the degree of loss for me in the interaction?

Based on our perception of the answers to these questions, we decide our future course of action in the interaction. In other words, not all the answers to the questions that we ask ourselves before initiating the interaction are addressed by the analysis of trust in the interaction. Those questions related with experiencing the level and magnitude of loss in the interaction are related and best expressed by the analysis of the risk in the interaction. But at the end it is the combination of the answers that we perceived for these questions, i.e. in other words the combination of the answers by the analysis of trust and risk in the interaction which affects the initiating agent in making an interaction decision accordingly. This fear of loss or not achieving what is desired is associated with 'Risk' in the interaction. Risk is an omnipresent factor in the modern world e-commerce interactions. It can easily be distinguished from other events due to the unwanted effects associated with it, and its ability to change the outcome of the interaction in a negative way. By specifying 'negative way' we mean the loss, or the 
occurrence of an undesired outcome in the interaction. In the literature, researchers have defined risk by associating it with an unbiased outcome [11]. But the reality is that an unbiased event might not change the outcome of the interaction that is 'unwanted' in the interacting agent view as much as the negative outcome does, and hence we consider that risk in an interaction is associated with the occurrence of negative outcomes in it. Risk analysis is important in the study of behavior in e-commerce interaction as there is a whole body of literature based in rational economics that argues that the decision to buy is based on the risk-adjusted cost-benefit analysis [12]. Risk plays a central role in deciding whether to proceed with a transaction or not. It can broadly be defined as an attribute of decision-making that reflects the variance of its possible outcomes. Thus, it commands a central role in any discussion that is related to a transaction.

To assess the possible Risk in an interaction carried out in a domain, it is first important to comprehend how risk is interpreted in that particular domain. In the literature there have been various definitions proposed by different researchers according to the context in which they are being discussed in. Those definitions are defined according to how it fits and best expresses its object of analysis in that particular domain. Similarly risk is assessed according to how it is defined in a particular domain. The definition of risk and its assessment method in a domain cannot be used to define and assess risk in other domains, as the way risk is interpreted and assessed in those varies, and hence would give in-correct conclusions if applied. So in order to address such issues, in our previous work we defined our interpretation of perceived risk according to its object of analysis in the domain of business interactions as 'the likelihood that the risk assessing agent might not achieve its desired outcomes of the interaction due to the risk assessed agent not acting as expected according to its expectations, in the given context and time once the interaction begins, resulting in the loss of investment and resources involved in the interaction'. As can be seen from the definition, we interpret perceived risk in the domain of e-business interactions as a multi-dimensional construct which is a combination of its sub-categories. Those subcategories are performance risk and financial risk in the interaction. Similarly the risk assessing agent has to assess and analyze these sub-categories while quantifying the level and magnitude of perceived risk in the interaction.

We define Performance risk in the interaction as the level and probability to which the risk assessing agent will not achieve its desired outcomes in interacting with a risk assessed agent. Desired outcomes are defined as the combination of the different assessment criteria which the risk assessing agent wants to achieve in the context of its interaction with the risk assessed agent. In order for the risk assessing agent to determine the performance risk in interacting with a risk assessed agent, we proposed the term FailureLevel and the Failure Scale. The Failure Scale represents the different levels of failure possible in an interaction. The FailureLevel quantifies the different levels of failure on the Failure Scale with a numerical value. In order to consider the dynamic nature of perceived risk, we consider that the risk assessing agent divides the time period of its interaction with the risk assessed agent in different non-overlapping 'time slots' and determines the FailureLevel of the risk assessed agent in each time slot. According to the time period of the risk assessing agent's interaction with the risk assessed agent, we have proposed different scenarios by which the risk assessing agent can determine the performance risk of the risk assessed agent according to the specific context and assessment criteria of its future interaction with it, in each time slot of its interaction. Interested readers are encouraged to refer to Hussain et al. [13] where the proposed methodology has been explained in detail. Due to space limitations we will not discuss the methodology in this paper. Based on the determined FailureLevel of the risk assessed agent in each time slot, the risk assessing agent determines the FailureLevel Curve (FLC) in interacting with the risk assessed agent. The FailureLevel Curve represents the performance risk of the risk assessed agent in committing to the specific context and assessment criteria of its interaction with the risk assessing agent. In other words, the FailureLevel Curve represents the probability of occurrence of different levels of failure from the Failure Scale in interacting with a risk assessed agent, according to its assessed behavior in the specific context and assessment criteria over the period of time of its interaction. An example of the FailureLevel Curve (FLC) of the interaction is shown in Figure 1.

Once the risk assessing agent determines the performance risk in interacting with the risk assessed agent then it should utilize it to determine the financial risk to it in interacting with that agent. In the domain of discussion of this paper, we consider that a risk assessed agent interacts with a risk assessing agent in order to give its desired outcomes, which it is capable of, in lieu to the pre-decided monetary financial value during the time period of interaction. Subsequently, the 


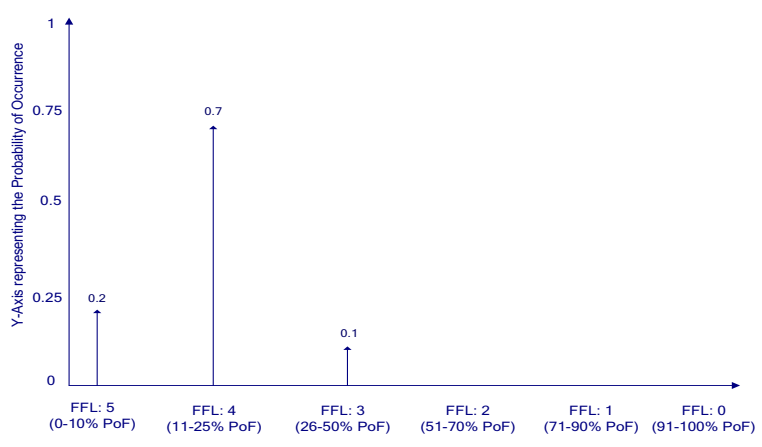

$X$-Axis showing the FailureLeve

Figure 1: The FailureLevel Curve of the Interaction

financial risk which the risk assessing agent has to determine in the interaction is in the monetary financial value that it invests and has at stake while interacting with the risk assessed agent. We define Financial risk in the interaction as the level to which the risk assessing agent will not achieve the full benefit of its invested financial resources in the interaction, and the level of un-served investment or the extra degree of financial resources that it has to keep at stake while interacting with the risk assessed agent, from its maximum investment capacity, due to its performance risk.

In order for the risk assessing agent to determine the financial risk in interacting with a risk assessed agent, we proposed in [14] that the risk assessing agent first ascertains its accurate investment in each time slot of its interaction with the risk assessed agent. Based on its accurate investment, the risk assessing agent ascertains the 'Amount Invested Curve' (AIC) of the interaction. The AIC represents the accurate level of the risk assessing agent's resources at stake in the interaction. It then determines the impact of the performance risk of the risk assessed agent on the AIC of the interaction, to determine the 'Factual Amount Invested Curve' (FAIC) of the interaction. The FAIC represents the actual level of the resources needed to be at stake in the interaction due to the performance risk of the risk assessed agent. The risk assessing agent based on its maximum investment capacity in the interaction then determines the 'Loss of Investment Probability' (LOIP) and 'Possible Consequences of Failure' (PCF) in interacting with the risk assessed agent, on the FAIC, as shown in Figure 2. The LOIP represents the probability to which the risk assessing agent will not get the maximum benefit of its invested resources in the interaction and the PCF represents the extra degree of financial resources that the risk

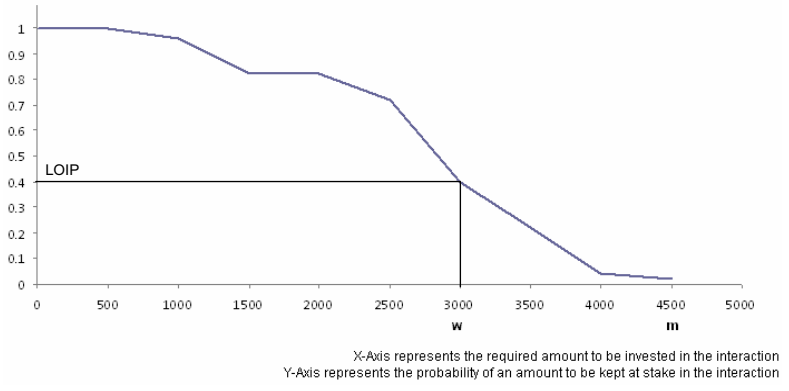

Figure 2: The Factual Amount Invested Curve of the Interaction

assessing agent has to keep at stake while interacting with the risk assessed agent. Interested readers are encouraged to look at Hussain et al. [14] where we explain in detail how the risk assessing agent determines the financial risk in forming an interaction with the risk assessed agent. Due to space limitations we will not discuss the methodology in this paper.

Once the risk assessing agent assesses and determines the subcategories of perceived risk, i.e. the performance risk and financial risk in forming an interaction with a risk assessed agent, it should then determine the level and magnitude of perceived risk in interacting with that agent. In this paper we will propose the methodology by which the risk assessing agent determines the numerical level and magnitude of perceived risk in forming an interaction with an agent, according to the different sub-categories of perceived risk determined in interacting with it. The proposed methodology is explained in the next sections.

\section{Ascertaining the Numerical Level and Magnitude of Perceived Risk}

The motive for ascertaining the different levels and magnitude of perceived risk in the interaction numerically is due to the fact that the risk assessing agent ascertains the perceived risk in interacting with a risk assessed agent, for better decision making before initiating an interaction with it. Hence, it is logical that there is uncertainty in the risk assessing agent's mind while determining the level of perceived risk as it is being determined in an interaction which is going to be held at a future period of time, in which nothing is certain. In other words, the perceived risk is being determined in an interaction which is yet to be carried out; and that interaction will have a specific outcome but there is uncertainty as to exactly which outcome will occur from the likely ones. This uncertainty which is present in the risk assessing agent's mind can be classified into two types: 'ambiguity' and 'vagueness'. 
As our aim in this paper is towards risk analysis, we define ambiguity as that uncertainty in the risk assessing agent's mind which represents its inability to identify the concrete level/s or magnitudes of perceived risk present in interacting with the other agent, whereas vagueness is defined as that type of uncertainty which represents its inability to identify the degree or likelihood to which those levels will occur in the interaction. In order to alleviate these types of uncertainties from the risk assessing agent's considerations, we propose that it calculate the different levels of perceived risk and their magnitude of occurrence in interacting with a risk assessed agent.

The subcategories which the risk assessing agent determines in order to ascertain the perceived risk in interacting with a risk assessed agent were mentioned earlier. But while ascertaining the perceived level of risk in an interaction, we propose that the risk assessing agent should take into consideration only two if its constituents from the determined subcategories, namely the possible consequences of failure and the loss of investment probability in the interaction. This is because the risk assessing agent considers the effect of the performance risk of the risk assessed agent while determining the financial risk in interacting with it. To avoid having to do this again and to prevent its duplicated impact, we propose that the risk assessing agent, while determining the perceived risk in interacting with the risk assessed agent, should consider only the possible consequences of failure and the loss of investment probability to its resources in interacting with that agent. So the inputs and output while determining the numerical and linguistic level of perceived risk in the interaction can be classified as:

\section{Inputs:}

- Possible Consequences of Failure to the risk assessing agent in interacting with the risk assessed agent (PCF);

- Loss in Investment Probability to the risk assessing agent in interacting with the risk assessed agent (LOIP).

\section{Output:}

- Level of Perceived Risk in the interaction (PR).

While ascertaining the numerical level of perceived risk in the interaction, the possibility distribution of each of the abovementioned constituents is utilized and not their probability distribution. This is to avoid the disadvantage of the probability distribution where the non-zero value must be assigned to an element from its given set or UoD, whose likelihood of occurrence is very high. Furthermore, whatever value is assigned to that element affects the probability value to be assigned to the other elements in the UoD, as the sum of all the probability distribution of the elements is constrained to a sum of 1 . In order to remove this disadvantage, we use possibility distribution to represent each constituent of risk in an interaction, as in this distribution assigning a likelihood of 1 to an element does not prevent the assigning of that likelihood to any other element from its UoD. Furthermore, the output, i.e. the magnitude of perceived risk in the interaction is also represented by possibility distribution which does not contain the disadvantages of the probability distributions mentioned earlier. The numerical magnitude of perceived risk in the interaction by using possibility theory is determined mathematically by a relation between:

Perceived Risk $=$ Possible Consequences of Failure $\mathrm{x}$ Loss of Investment Probability

The operator ' $\mathrm{x}$ ' between the two inputs represents convolution. In order to determine the distribution of each input constituents (PCF and LOIP) and the output function (PR), we first define the scope or the universe of discourse (UoD) within which each of the particular variable exists by the following sets:

Possible Consequences of Failure $(P C F)=\{0,1,2$, $3 \ldots \ldots . .100\}$ where each element has a unit of $\%$.

Loss of Investment Probability $($ LOIP $)=\{0,1,2$, $3 \ldots \ldots \ldots . . .100\}$ where each element has a unit of $\%$.

Perceived Risk $(P R)=\{0,1,2,3 \ldots \ldots \ldots .100\}$ where each element has a unit of $\%$.

To obtain the possibility distribution of an input variable, the likelihood of occurrence of each element from its universe of discourse should be determined. This likelihood of occurrence of an element is termed the 'degree of evidence' of its outcome, represented by ' $m$ (A)', where ' $A$ ' is an element from the universe of discourse of the input variable ' $X$ '. From the universe of discourse, those elements with degree of evidence greater than zero are called the 'focal elements' for the particular input variable. These elements represent the sets from the UoD for that variable upon which the evidence of occurrence focuses and which furthermore will be utilized from that input variable to determine the magnitude of perceived risk in the interaction. The degree of evidence of an element from the UoD should be in the interval between $[0,1]$ and the cumulative sum of the degree of evidence of all the focal elements from the UoD should satisfy the condition [15]:

$$
\sum_{A \in X} m(A)=1
$$

Equation 1 
where ' $A$ ' represents the focal elements belonging to the input variable ' $\mathrm{X}$ ',

$m$ (A) represents the degree of evidence of the focal element.

The possibility of an element 'A i' of the input variable $X$ can be determined from the focal elements of its UoD by [15]:

$$
\pi\left(A_{i}\right)=\sum_{j=1}^{n} m(A j)
$$

where $m\left(A_{j}\right)$ represents the degree of evidence of ' $A j$ ' from the focal elements of the input variable from its UoD, and which have been ordered such that $\mathrm{i}<\mathrm{j}$ and $\pi\left(\mathrm{A}_{\mathrm{i}}\right) \geq \pi\left(\mathrm{A}_{\mathrm{j}}\right)$.

In other words, $m$ (A) represents the degree of evidence that an element ' $A$ ' belongs exactly to ' $X$ ', the Belief that an element belongs to ' $\mathrm{X}$ ', Bel (A) represents the total evidence that the element belongs to ' $\mathrm{X}$ ' as well as to any subset of ' $\mathrm{X}$ '. Plausibility, $\mathrm{Pl}$ (A) is the total evidence that the element belongs to ' $\mathrm{X}$ ' as well as to any subset of ' $\mathrm{X}$ ', plus additional evidence for sets that overlap with ' $\mathrm{X}$ '. Belief / Plausibility measures are referred to as Necessity (N) / Possibility ( $\Pi$ ) measures respectively [15]. Possibility measures can be represented by a possibility distribution function:

$$
\Pi(X)=\max \{\pi(a) \mid a \in X\} \quad \text { Equation } 3
$$
where $\pi$ (a) is the possibility of the element ' $a$ '.

Equation 3 is repeated for each focal element of ' $\mathrm{X}$ ' to determine the possibility of its occurrence. Once the focal elements of the input variables (PCF and LOIP) along with their degree of evidence from their UoD have been determined, they must then be convolved to determine the perceived risk in the interaction. The focal elements of the output variable function from its defined universe of discourse are determined in the convolution process. The convolution of the possibility distributions is the artesian product of the input variables [15]. The convolution of the focal elements from the input $\mathrm{UoD}(\mathrm{X}$ and $\mathrm{Y})$ is done by taking their artesian products and is represented by:

$\mathrm{X} x \mathrm{Y}=\{<\mathrm{x}, \mathrm{y}>$ : where $\mathrm{x} \in \mathrm{X}$ and $\mathrm{y} \in \mathrm{Y}\}$ Equation 4 where $<\mathrm{x}, \mathrm{y}>$ denotes the tuple which represents the artesian product of the input focal elements from their UoD.

The possibility distribution of the focal elements of the resultant output variable as the result of the convolution of the inputs variables is represented by: $\pi(\mathrm{u})=\max \left\{\min \left[\pi_{\mathrm{x}}(\mathrm{x}), \pi_{\mathrm{Y}}(\mathrm{y})\right]\right\}$

Equation 5 where $u$ is the focal element of the output function determined as the artesian product of the inputs $\mathrm{f}(\mathrm{x}$, y), $\pi(u)$ is the possibility of focal element ' $u$ ' from the output universe of discourse.

The above equation gives the possibility of occurrence of the focal elements of the magnitude of perceived risk in the interaction due to the convolution of the focal elements of the inputs, the possible consequences of failure and the loss of investment probability. From the determined focal elements, the possibility distribution of the perceived risk in the interaction can be ascertained. In the next section, we will explain the steps by which the risk assessing agent by using the possibility theory can ascertain the focal elements and their degree of evidence from the UoD of PCF and LOIP to determine their possibility distributions.

\section{Determining the Possibility Distributions of Input Constituents}

In order to ascertain the numerical level of perceived risk in the interaction by using possibility distribution, the risk assessing should first identify the focal elements along with their degree of evidence of the inputs from their universe of discourse which range from $\{0 \ldots 100\}$. As discussed in the previous section, both the constituents, namely the loss of investment probability (LOIP) and the possible consequences of failure (PCF) in interacting with the risk assessed agent are determined from the Factual Amount Invested Curve (FAIC) in interacting with it as shown in Figure 2. To determine the focal elements and the possibility distribution of the PCF in the interaction, the risk assessing agent from its maximum investment capacity should determine the levels of extra financial resources that it has to keep at stake or the levels of un-served investment in the interaction while interacting with the risk assessed agent. To achieve that:

- The risk assessing agent should determine the probability mass function (PMF) of the FAIC in interacting with the risk assessed agent. The PMF of the FAIC shows the probability of an amount from the required resources that the risk assessing agent has to keep at stake throughout the duration of interacting with the risk assessed agent.

- It should then determine the point on the PMF of the FAIC which represents its maximum investment capacity in the interaction, which is termed as ' $x$ '.

- From point ' $x$ ' the risk assessing agent should determine the degrees of extra financial resources that it has to keep at stake or the levels of its un-served investment in the interaction.

- From the UoD for the PCF, the focal elements should be chosen according to the measure of step size 
on the FAIC of the levels of un-served investment in the interaction.

- The risk assessing agent should then determine the degree of evidence $(\mathrm{m}(\mathrm{A}))$ of each focal element which represents the level of un-served investment in the interaction. This is determined by taking into consideration the PMF of the particular financial amount from the FAIC and then converting it to possibility distribution, satisfying equation 1 .

- Based on the degree of evidence calculated for each focal element from the UoD, the possibility distribution of the PCF can be determined by using equation $2-3$.

- The LOIP of the interaction in contrast to the PCF is a single crisp value in the range of [0-1], which shows the ordinate on the FAIC at the end of its maximum investment capacity in the interaction. But in order to utilize a unified and comparable numerical scale to the two inputs, the range of the LOIP is normalized in the range between 0-100.

- Hence, the ordinate of the FAIC after point ' $x$ ' is taken as the focal element from its UoD to represent the LOIP in the interaction. The degree of evidence of the focal element is taken as 1 .

Once the focal elements and their degree of evidence for each input variable have been determined, the risk assessing agent should then convolve them to determine the focal elements of perceived risk in the interaction from its UoD by using equation 4. The possibility of occurrence of the focal elements of perceived risk in the interaction can then be determined by using equation 5 . In the next section, we will explain with an example the process of the risk assessing agent determining the numerical level of perceived risk in an interaction with the risk assessed agent by using possibility theory.

\section{Determining the Numeric Level and Magnitude of Perceived Risk}

To determine the numerical level of perceived risk by using possibility theory, let us consider an interaction scenario where the risk assessing agent ' $A$ ' wants to determine the level and magnitude of perceived risk before making an interaction-based decision with agent ' $\mathrm{B}$ '. Further let us assume that the risk assessing agent ' $A$ ' determines the performance risk in interacting with agent ' $\mathrm{B}$ ' according to its desired outcomes in the interaction as shown in Figure 1. It then determines its impact on the AIC of its interaction to ascertain the Factual Amount Invested Curve (FAIC) of its interaction as shown in Figure 2. Let us consider that the maximum investment capacity of the risk assessing agent ' $A$ ' in the interaction is $\$ 3,000$. Based on its maximum investment capacity in the interaction the risk assessing agent can determine the PCF and LOIP in interacting with the risk assessed agent. But in order to determine the focal elements and their degree of evidence from the UoD of the input variable, the risk assessing agent has to determine the level and magnitude of un-served investment in interacting with the risk assessed agent. It can do that by executing the steps mentioned in the previous section to identify the focal elements of the PCF of the interaction, along with their degree of evidence to transform it into a possibility distribution. The focal elements from the UoD of the input variable PCF are represented in Figure 3.

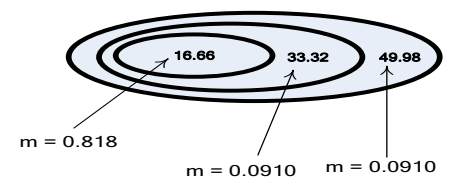

Figure 3: The focal elements and their degree of evidence for the input: Possible Consequences of Failure

Similarly, the focal elements from the UoD of the input variable the LOIP in the interaction by using the steps mentioned in the previous section are represented in Figure 4.

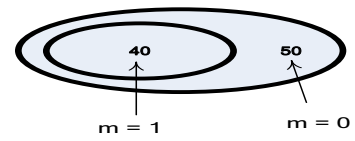

Figure 4: The focal elements and their degree of evidence for the input: Loss of Investment Probability in an Interaction

Once the focal elements and their degree of evidence for the input constituents PCF and the LOIP have been determined, then the risk assessing agent ' $A$ ' by equation 4 should convolve them to obtain the focal elements of the perceived risk in interacting with agent ' $\mathrm{B}$ '.

PCF X LOIP $=$

$\{<49.98,50>,<49.98,40>,<33.32,50>,<33.32,40>$, $<16.66,50>,<16.66,40>\}$

The artesian product of PCF and LOIP by combining the elements from their UoD along with their possibility is represented in Table 1. The degree of evidence for the focal elements representing the magnitude of perceived risk in the interaction is determined by using equation 5 . 


\begin{tabular}{|c|c|c|}
\hline $\begin{array}{c}\text { Artesian } \\
\text { product of the } \\
\text { inputs }\end{array}$ & $\begin{array}{c}\text { Resultant } \\
\text { Focal element }\end{array}$ & $\begin{array}{c}\text { Possibility of } \\
\text { occurrence of the } \\
\text { Focal elements }\end{array}$ \\
\hline$<49.98,50>$ & 99.98 & 0 \\
\hline$<49.98,40>$ & 89.98 & 0.0910 \\
\hline$<33.32,50>$ & 83.32 & 0 \\
\hline$<33.32,40>$ & 73.32 & 0.182 \\
\hline$<16.66,50>$ & 66.66 & 0 \\
\hline$<16.66,40>$ & 56.66 & 1 \\
\hline
\end{tabular}

Table 1: The focal elements and their possibilities for the output variable: Perceived Risk

Figure 5 shows the possibility distribution of the perceived risk in the risk assessing agent's ' $A$ ' interaction with agent ' $\mathrm{B}$. This distribution also shows the different levels of perceived risk that could be present along with the possibility or likelihood of occurrence of those levels.

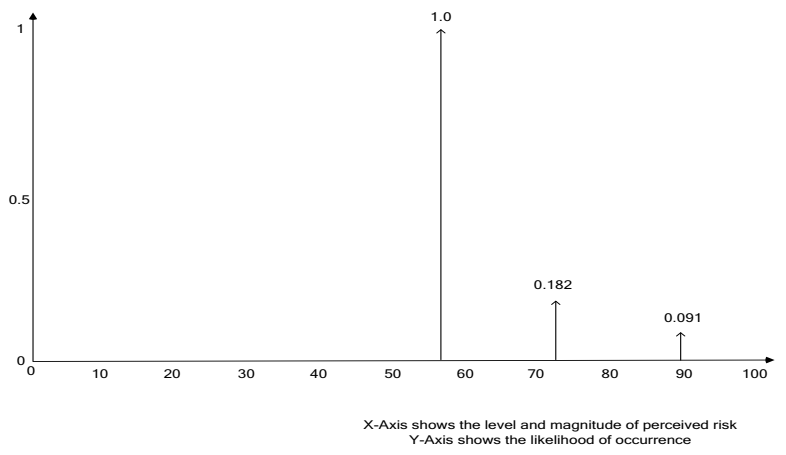

Figure 5: The possibility distribution of Perceived Risk in the Interaction

From the above example, it can be concluded that:

- Only those elements from the UoD of an input variable which have a positive degree of evidence (i.e. which are the focal element from that input variable) have an effect on the output set during convolution.

- During the process of convolution of the input variables to determine the focal elements of perceived risk, if the artesian product of the two input variables results in an element which is greater than 100, then we consider the artesian product of those elements as 100 , as the UoD of the output variable ranges from [0...100].

- The obtained numerical level of perceived risk is a result of its various constituents determined by the risk assessing agent in interacting with a risk assessed agent.

By using the proposed approach the risk assessing agent can determine the levels of perceived risk and their magnitude of occurrence in forming an interaction with a risk assessed agent. Also by utilizing the focal elements and their degree of evidence of each input variable, the risk assessing agent can determine semantically the level of perceived risk or the linguistic level of perceived risk in forming an interaction with a risk assessed agent. It can then utilize the determined level of perceived risk to manage it according to its risk propensity nature or risk taking attitude, and based on that make an informed interaction-based decision with that agent. This is our future work.

\section{Conclusion}

In this paper we proposed a methodology by which the risk assessing agent can ascertain the different levels and magnitude of perceived risk in forming an interaction with the risk assessed agent. We utilized the possibility theory to achieve that. By determining the different levels and magnitude of perceived risk, the risk assessing agent can get an idea of how its interaction with the risk assessed agent will proceed, if it chooses that agent to interact with. Also it can utilize each level of perceived risk and its magnitude of occurrence while making an informed interactionbased decision with that agent, according to its risk propensity or risk raking nature.

\section{References}

[1] Elizabeth Chang, Tharam Dillon, and F. K. Hussain, Trust and Reputation for ServiceOriented Environments. West Sussex, England: John Wiley \& Sons, Ltd, 2006.

[2] Tom O'Reilly, "What is Web 2.0," 2005.

[3] Australian Bureau of Statistics, "Online Shopping Blooms," in Use of the Internet by Householders, Australia, Nov 2000, 2000

[4] Thuiswinkel Organisatie, "Internet shopping gaining popularity in the Netherlands," in $m \& c, 2007$.

[5] Reineke Reitsma, "European Online Shopping Landscape 2007," in Forrester Research, 2007.

[6] Anwitaman Datta, Manfred Hauswirth, and Karl Aberer, "Beyond "web of trust": Enabling P2P E-commerce," in IEEE International Conference on E-Commerce (CEC'03). Newport Beach, CA, USA: IEEE Computer Society, 2003, pp. 303-312.

[7] Audun Jøsang, Claudia Keser, and T. Dimitrakos, "Can We Manage Trust?," in Third International Conference on Trust Management (iTrust 2005), vol. LNCS 3477. Rocquencourt, France: Springer 2005, pp. 93-107.

[8] Farookh Khadeer Hussain, Elizabeth Chang, and Tharam S. Dillon, "Trustworthiness and CCCI metrics in P2P communication," Journal of Computer Systems: Science \& Engineering, vol. 19, pp. 173-190, 2004.

[9] Karl Aberer and Z. Despotovic, "Managing trust in a Peer-2-Peer Information System," in Proceedings of the Tenth International Conference on Information and Knowledge Management (CIKM'01), ACM, Ed. Atlanta, Georgia, USA, 2001, pp. 310 317.

[10] Lewis Hassell, "Affect and Trust," in Third International Conference on Trust Management (iTrust'05), vol. LNCS 3477, Springer, Ed. France: Springer, 2005, pp. 131145 .

[11] R.C. Mayer, J.H. Davis, and F. D. Schoorman, "An Integrative Model of Organizational Trust," The Academy of Management Review, vol. 20, pp. 709-734, 1995.

[12] Sander Greenland, "Bounding Analysis as an Inadequately Specified Methodology," Risk Analysis, vol. 24, pp. 1071-1083, 2004

[13] Omar Khadeer Hussain, Elizabeth Chang, Farookh Khadeer Hussain, and Tharam S. Dillon, "A methodology to quantify failure for risk-based decision support system in digital business ecosystems," Data \& Knowledge Engineering, vol. 63, pp. 597-621, 2007.

[14] Omar Hussain, Elizabeth Chang, Farookh Hussain, and Tharam Dillon, "Towards Quantifying the Possible Risk in e-commerce Interactions for RDSS," in Proceedings of the IEEE International Conference on e-Business Engineering (ICEBE'07), IEEE, Ed. Hong Kong, China: IEEE, 2007, pp. 89-96.

[15] Didier Dubious, Henri Prade, Henri Farreny, Roger Martin-Clouaire, Claudette Testemale, and E. F. Harding, Possibility Theory: An Approach to Computerized Processing of Uncertainty. New York: Plenium Press, 1988. 